\title{
A UTILIZAÇÃO DE ALUMINO-SILICATOS COMO AGENTES PROTETORES CONTRA A AFLATOXICOSE NA ALIMENTAÇÃO DE FRANGOS DE CORTE' ${ }^{1}$
}

\author{
MORAIS, S.A; SILVA, R.D.M. \\ Departamento de Zootecria - ESALO/USP - C.P. 9, CEP: 13418-96a-Plracicaba,SP. \\ FONSECA, H.; DOMINGUES, M.A.C. \\ Departamento de Clencia e Tecnologia Agrolindustrial - ESALO/USP - C.P. 9, CEP: 13418-960-Piracicaba,SP.
}

\begin{abstract}
RWSUMO: O experimento foi conduxido para testar a eficilncia de dois alumino-silicatos, no controle da aflatoxicose em frangos de corte (machos). Foram efetuadas anflises de sangue para verificafilo da infulencia da afiatoxina no perfil sanguineo das aves. Os resultados mostraram efeito negativo $(\mathrm{P}<0,05)$ da afiatoxina no desempenho das aves no que se refere ao peso corporal, ganho de peso, consumo e conversäo alimentares, quando comparados com o controle. Os niveis sanguineos de proteina total se apresentaram reduxidos $(P<0,05)$ e os da transaminase glutamica oxalactica aumentados $(\mathrm{P}<0,05)$ em relaça a controle. Os níveis da fosfatase alcalina, tempo de protrombina, gama-glutamil transferase, bilirrubina e da capacidade totol de ligacho do ferro nilo apresentaram diferencas significativas. Os alumino-silicatos nåo neutralizaram os efeitos da afiatoxina sobre as aves.

Descritares: aflatoxina, alumino-silicatos, franges de corte, pardmetros sangufneos.
\end{abstract}

\section{THE USE OF ALUMINOSIICATES IN BROLRR FJRDNG AS PROTBCTOR AGUNIS AGAINST AFLATOXICOSBS}

ADsTraCT: This experiment was conducted to evaluate the eficiciency of two alumino-silicates against deleterions effects of a toxin on growth performance and blood parameters of male broilers. The products did not counteract the negative effects on growth performance. Total protein and glutamic-oxalacetic transaminase in blood from biris fed contaminated and non contaminated rations, were negatively affected $(P<0.05)$ by the toxin. Other parameters like alkaline phosphatase, prothrombin time, gamma ghtamyl transferase, bilinubin and total iron binding capacity did not show significant differences when compared to the control.

Key Worls: aflatoxin, alumino-silicates, broiler, blood parameters.

\section{INTRODUÇÃO}

As aflatoxinas constituem um grupo de micotoxinas produzidas por dois fungos: Aspergillus flavus e Aspergillus parasiticus. 0 Aspergillus flavus tipicamente produz aflatoxina B1 e B2 e o Aspergillus parasiticus isolado produz $G 1$ e G2, bem como B1 e B2 (DIENER et al., 1987). Dos 4 grupos de aflatoxina a Bı é a mais tóxica conhecida pelo homem e produz tumores em todas as espécies de animais estudadas (NYATHI et al., 1985 e DALVI, 1986).

A ôcorrência da perda de peso corporal e redução do consumo alimentar é característica de aves com aflatoxicose (HUFF et al., 1988). Desta forma para evitar os prejuizos econômicos ocasionados por essas micotoxinas são utilizados vários métodos físicos e químicos para remoção da aflatoxina dos alimentos. Dentre eles destaca-se a utilização de bases, tais como, hidróxido de sodio e de cálcio, as quais são efetivas mas relativamente caras para remoção da toxina em produtos contaminados (DOLLEAR et al., 1968). A utilização de amônia para desentoxicar alimentos contaminados para aves através da inativação da aflatoxina não surtiu efeito positivo (HUGGES et al., 1979). Entretanto, segundo PHILLIPS et al. (1988) o emprego dos alumino-silicatos e argila de bentonita, causaram redução acentuada dos efeitos negativos da aflatoxina em aves.

$O$ objetivo da presente pesquisa foi o de estudar a eficácia do alumino-silicato na proteção

\footnotetext{
1 Este trabalho é parte da dissertação do primeiro autor apresentado para obtençāo do título de Mestre em Agronomia, área de concentração Ciência Animal e Pastagens.
} 
às aves contra a ação negativa da aflatoxina, analisando o seu efeito sobre o desempenho relativo ao peso corporal das aves, consumo alimentar, ganho de peso, conversão alimentar, mortalidade e nos parâmetros sanguíneos: proteína total, fosfatase alcalina, tempo de protrombina, bilirrubina e capacidade total de ligação do ferro.

\section{MATERIAL E MÉTODOS}

Foram utilizados 600 pintos machos de corte da linhagem Hubbard-Peterson. Os pintos foram pesados em grupos de 20 e distribuidos ao acaso dentro de cada box do galinheiro experimental.

O delineamento experimental foi inteiramente casualizado, constituido de 6 tratamentos: 1- Sem adição de alumino-silicato e sem aflatoxina $B_{1}$ (controle). 2- Sem adição de alumino-silicato e $428,8 \mathrm{ppb}$ de aflatoxina B1. 3Adição de $0,75 \%$ de alumino-silicato $1^{\prime}$ e 454,6 ppb de aflatoxina B1. 4- Adição de 1,50\% de alumino-silicato 1 e 350,2 ppb de aflatoxina B1. 5Adição de $0,25 \%$ de alumino-silicato $2^{2}$ e 497,0 ppb de aflatoxina B1. 6- Adição de $0,50 \%$ de alumino-silicato 2 e 381,7 ppb de aflatoxina B1.

Cada tratamento era constituido de 5 repetições. O período experimental foi de 47 dias.

O programa de alimentação constou de ração corte inicial ao longo de todo experimento. Os niveis nutritivos utilizados foram os recomendados para a linhagem adquirida $\mathrm{e}$ a alimentação foi fornecida à vontade.

A composição das dietas utilizadas no experimento é mostrada na TABELA 1.

A extração da toxina do farelo de amendoim contaminado e da ração foi realizada pelo método de PONS modificado de acordo com FONSECA $(1991)^{3}$, e a quantificação do farelo pelo método de COOMES \& FEUELL (1965) e da ração pela cromatografia de camada delgada utilizando o Fotodensitômetro CAMAG TLC SCANNER II.

O nível total de aflatoxina em cada ração é resultado da somatória das aflatoxinas $\mathrm{B}_{2}, \mathrm{G}_{1}, \mathrm{G}_{2}$ e B1.

\footnotetext{
${ }^{1}$ Bentonit União Nordeste S.A. - São Paulo,SP.

${ }^{2}$ Anitox Corporation - Buford, Georgia, E.U.A.

${ }^{3}$ FONSECA, H. (ESALQ, Laboratório de Micotoxinas), Comunicação pessoal, 1991.
}

Segundo CARNAGHAN et al. (1963), aflatoxina $\mathrm{G} 1$ corresponde a $50 \%$ da $\mathrm{B1}, \mathrm{B} 2$ corresponde a $50 \%$ da $\mathrm{G} 1$ e $\mathrm{G} 2$ corresponde a $50 \%$ da B2. Desta forma, a TABELA 2 mostra o teor total do equivalente em aflatoxina $B 1$.

Os lotes foram pesados semanalmente, do início ao fim do experimento. $O$ consumo de ração foi determinado semanalmente para se obter a conversão alimentar. A mortalidade foi registrada diariamente e as aves mortas eram pesadas logo após a observação do evento. As análises de sangue foram realizadas no Laboratório Clínico São Lucas, localizado em Piracicaba, São Paulo.

Os valores médios das parcelas experimentais referentes ao desempenho foram calculados pelo procedimento PROC ANOVA (SAS INSTITUTE, 1987), para dados balanceados. Os valores médios referentes aos parâmetros sanguíneos foram calculados pelo procedimento PROC GLM (SAS INSTITUTE, 1987) para dados desbalanceados devido a existência de parcela perdida. As médias foram submetidas ao teste de DUNNETT, que foi usado com o objetivo de testar cada tratamento dentro de cada período com controle (GILL, 1986).

\section{RESULTADOS E DISCUSSÃO}

Os efeitos dos tratamentos sobre peso corporal médio das aves estão mostrados na TABELA 3.

O teste de médias mostrou uma redução significativa do peso corporal dos frangos em relação ao controle quando as aves recebiam os tratamentos com adição de farelo de amendoim na presença ou ausência dos alumino-silicatos c aflatoxina. Os resultados estão concordantes com os trabalhos de HUFF et al. (1986a e 1986b), MERKLEY et al. (1987), RAM et al. (1988).

Os resultados referentes ao ganho de peso apresentados na TABELA 4 mostraram, com exceção das aves que recebiam ração sem adição de alumino-silicatos 1 e na presença de aflatoxina, redução significativa quando comparados com o controle nos períodos de $0-7,8-14,15-21,22-28$ e 29-35 dias de vida em aves tratadas com as rações 3, 4, 5 e 6. Entretanto, nos dois períodos finais não foi verificada redução significativa $\mathrm{em}$ relação ao controle. Esses resultados concordam com os trabalhos de COTTIER et al. (1968). No entanto, os alumino-silicatos 1 e 2 neste trabalho não apresentaram tanta eficiência quanto ao aluminosilicato citado na literatura por KUBENA et al. (1990) em frangos. 
TABELA 1 - Composição percentual das rações.

\begin{tabular}{|c|c|c|c|c|c|c|}
\hline \multirow[t]{2}{*}{ COMPOSIÇÃO } & \multicolumn{6}{|c|}{ RAÇÕES (Tratamentos) \% } \\
\hline & 1 & 2 & 3 & 4 & 5 & 6 \\
\hline Milho & 61,60 & 63,70 & 62,80 & 61,90 & 63,40 & 63,10 \\
\hline F. Soja & 32,80 & - & - & - & - & - \\
\hline F. Amendoim & - & 30,00 & 30,00 & 30,00 & 30,00 & 30,00 \\
\hline F. Carne & 2,99 & 2,99 & 3,18 & 3,36 & 3,05 & 3,11 \\
\hline Calcáreo & 0,53 & 0,83 & 0,80 & 0,78 & 0,82 & 0,81 \\
\hline Fosf. Bicalc. & 1,16 & 1,02 & 0,97 & 0,91 & 1,00 & 0,98 \\
\hline DL-Met. & 0,21 & 0,28 & 0,28 & 0,28 & 0,28 & 0,28 \\
\hline $\mathrm{L}-\mathrm{Lis}$. & - & 0,48 & 0,48 & 0,47 & 0,48 & 0,48 \\
\hline S. Vit. & 0,40 & 0,40 & 0,40 & 0,40 & 0,40 & 0,40 \\
\hline S. Min. & 0,10 & 0,10 & 0,10 & 0,10 & 0,10 & 0,10 \\
\hline Sal & 0,25 & 0,25 & 0,25 & 0,25 & 0,25 & 0,25 \\
\hline Alum. Sil. 1 & - & - & 0,75 & 1,50 & - & - \\
\hline Alum. Sil. 2 & - & - & - & - & 0,25 & 0,50 \\
\hline Total & 100 & 100 & 100 & 100 & 100 & 100 \\
\hline
\end{tabular}

TABELA 2 - Teor das rações em equivalência de aflatoxina $\mathbf{B}_{1}$.

\begin{tabular}{|c|c|c|c|c|c|c|}
\hline \multirow[b]{2}{*}{ TOXINA } & \multicolumn{6}{|c|}{ RACÕES } \\
\hline & 1 & 2 & 3 & 4 & 5 & 6 \\
\hline \multicolumn{7}{|c|}{$\mathrm{ppb}$} \\
\hline B1 & ND & 338,0 & 367,0 & $272 ; 0$ & 345,0 & 286,0 \\
\hline B2 & ND & 21,8 & 20,0 & 19,2 & 23,5 & 19,0 \\
\hline G1 & ND & 62,5 & 63,5 & 54,5 & 120,5 & 73,5 \\
\hline $\mathrm{G} 2$ & ND & 6,5 & 4,1 & 4,5 & 8,0 & 3,2 \\
\hline TOTAL & ND & 428,8 & 454,6 & 350,2 & 497,0 & 381,7 \\
\hline
\end{tabular}


TABELA 3 - Efeito dos tratamentos sobre o peso corporal das aves em função do tempo.

\begin{tabular}{ccccccccc}
\hline TRATAMENTO & \multicolumn{7}{c}{ PESO CORPORAL (g) } \\
\hline & 0 & 7 & 14 & 21 & 28 & 35 & 42 & 47 \\
& & & & (dias de idade) & & & \\
\hline 1 & $44^{\natural}$ & $139^{\natural}$ & $346^{\natural}$ & $665^{\natural}$ & $1063^{\natural}$ & $1543^{\natural}$ & $2009^{\natural}$ & $2381^{\natural}$ \\
2 & $44^{\natural}$ & $118^{b}$ & $268^{b}$ & $515^{b}$ & $833^{b}$ & $1251^{b}$ & $1688^{b}$ & $2016^{b}$ \\
3 & $43^{\natural}$ & $120^{b}$ & $269^{b}$ & $515^{b}$ & $842^{b}$ & $1251^{b}$ & $1732^{b}$ & $2045^{b}$ \\
4 & $44^{\natural}$ & $118^{b}$ & $272^{b}$ & $513^{b}$ & $834^{b}$ & $1287^{b}$ & $1721^{b}$ & $2062^{b}$ \\
5 & $43^{\natural}$ & $112^{b}$ & $255^{b}$ & $496^{b}$ & $823^{b}$ & $1248^{b}$ & $1725^{b}$ & $2096^{b}$ \\
6 & $44^{a}$ & $122^{b}$ & $244^{b}$ & $469^{b}$ & $779^{b}$ & $1188^{b}$ & $1614^{b}$ & $1919^{b}$ \\
\hline \hline
\end{tabular}

TABELA 4 - Efeito dos tratamentos sobre o ganho de peso das aves em cada um dos períodos estudados.

\begin{tabular}{|c|c|c|c|c|c|c|c|}
\hline \multirow[t]{2}{*}{ TRATAMENTO } & \multicolumn{7}{|c|}{ GANHO DE PESO (g) } \\
\hline & $0-7$ & $8-14$ & $15-21$ & $22-28$ & $29-35$ & $36-42$ & $43-47$ \\
\hline \multicolumn{8}{|c|}{ (dias de idade) } \\
\hline 1 & 95 & $207^{\circ}$ & $319^{\circ}$ & $397^{\circ}$ & $480^{\circ}$ & $465^{m}$ & $372^{\circ}$ \\
\hline 2 & $74^{6}$ & $150^{b}$ & $244^{b}$ & $319^{b}$ & $418^{b}$ & $436^{\circ}$ & $327^{\circ}$ \\
\hline 3 & $77^{b}$ & $148^{b}$ & $245^{b}$ & $327^{\circ}$ & $408^{b}$ & $481^{\circ}$ & $313^{a}$ \\
\hline 4 & $74^{b}$ & $153^{b}$ & $241^{b}$ & $320^{b}$ & $453^{b}$ & $434^{*}$ & $340^{\circ}$ \\
\hline 5 & $69^{b}$ & $142^{b}$ & $240^{6}$ & $327^{\circ}$ & $424^{b}$ & $476^{\circ}$ & $371^{*}$ \\
\hline 6 & $78^{b}$ & $122^{b}$ & $224^{b}$ & $310^{6}$ & $408^{b}$ & $425^{\prime \prime}$ & $305^{n}$ \\
\hline
\end{tabular}

Valores em uma mesma coluna com diferentes sobrescritos, diferem significativamente do controle $(\mathrm{P}<0,05)$.

A TABELA 5 mostra o efeito dos tratamentos sobre o consumo alimentar.

$O$ teste de médias mostra que a aflatoxina causou redução significativa do consumo das aves somente nos períodos de 0-7, 22-28, 29-35 e 36-42 dias de vida, em relação ao controle.

Nos períodos de 8-14, 15-21 e 43-47 dias de vida existiu redução do consumo, mas esta não Sci. agric., Piracicaba, 50(2):311-320, jun./set., 1993 foi estatisticamente significativa. 0 tratamento contendo farelo de amendoim com adição de alumino-silicato 1 ao nível de $0,75 \%$ causou redução significativa do consumo nos períodos de $0-7,8-14,21-28,29-35,36-42$, $43-47$ dias de vida, no entanto, nos períodos de 15 - 21 dias de vida a redução não foi significativa. 
TABELA 5 - Efeito dos tratamentos sobre o consumo alimentar das aves em cada um dos periodos estudados.

\begin{tabular}{|c|c|c|c|c|c|c|c|}
\hline \multirow[t]{3}{*}{ TRA } & \multicolumn{7}{|c|}{ CONSUMO ALIMENTAR (g) } \\
\hline & 0.7 & $8-14$ & $15-21$ & $22-28$ & $29-35$ & $36-42$ & 43-47 \\
\hline & \multicolumn{7}{|c|}{ (dias de idade) } \\
\hline 1 & $2842^{\circ}$ & $6420^{\circ}$ & $9688^{\circ}$ & $15326^{\circ}$ & $19044^{*}$ & $19298^{\curvearrowleft}$ & $15176^{\circ}$ \\
\hline 2 & $2414^{\mathrm{b}}$ & $5546^{a}$ & $8078^{\circ}$ & $10304^{b}$ & $13902^{b}$ & $15214^{b}$ & $12172^{a}$ \\
\hline 3 & $2280^{\circ}$ & $5436^{b}$ & $7702^{a}$ & $10256^{b}$ & $14272^{b}$ & $15064^{b}$ & $11562^{b}$ \\
\hline 4 & $2372^{6}$ & $5554^{\circ}$ & $8268^{*}$ & $12578^{b}$ & $16532^{\circ}$ & $17438^{a}$ & $12506^{\circ}$ \\
\hline 5 & $2444^{b}$ & $5558^{a}$ & $8002^{a}$ & $11638^{b}$ & $14850^{b}$ & $15974^{b}$ & $12148^{\circ}$ \\
\hline 6 & $2180^{\circ}$ & $5372^{b}$ & $7342^{b}$ & $10992^{b}$ & $14894^{b}$ & $15736^{b}$ & $11214^{b}$ \\
\hline
\end{tabular}

Valores em uma mesma coluna com diferentes sobrescritos, diferem significativamente do controle $(\mathrm{P}<0,05)$.

Foi verificada redução significativa do consumo nos períodos de 0-7 e 22-28 dias de vida nas aves que se alimentaram de ração com adição de $1,50 \%$ de alumino-silicato 1 e 350,2 ppb de aflatoxina B1, mas não foi verificada nos períodos de $8-14,15-21,29-35,36-42$ e 43-47 dias de vida das aves.

As aves que consumiram a ração com adição de $0,25 \%$ de alumino-silicato 2 e $497,0 \mathrm{ppb}$ de aflatoxina $B 1$ reduziram significativamente $o$ consumo alimentar nos períodos de 0-7, 22-28, 29 35 e 36-42 dias, porém a redução não foi significativa nos períodos de 8-14, 15-21, 43-47 dias. Foi, também, observada redução significativa no consumo alimentar em relação ao controle quando as aves foram alimentadas com ração contendo $0,50 \%$ de alumino-silicato 2 e $381,7 \mathrm{ppb}$ de aflatoxina $B 1$ nos períodos de 0-7, 8-14, 15-21, 22-28, 29-35, 36-42 e 43-47 dias. Esses resultados estão concordantes com os obtidos por OSTROWISKI-MEISSNER (1983).

Os valores de conversão alimentar mostrados na TABELA 6 demonstram que as aves que receberam rações com adição do farelo de amendoim na presença ou ausência dos aluminosilicatos e aflatoxina com exceção feita para o tratamento com adição de $1,50 \%$ de aluminosilicato 1 e 350,2 ppb de aflatoxina B1 no período de 8-14 dias, não pioraram de maneira significativa em relação ao controle. Entretanto, a literatura mostra que HUFF et al. (1986a e 1986b) e MERKLEY et al. (1987) observaram piora na conversão alimentar das aves que receberam rações contendo aflatoxina.

Na TABELA 7 são mostrados os números médios de aves mortas sob o efeito dos diferentes tratamentos.

Através dos resultados do teste de médias, pode-se verificar que não existiu diferença significativa do número médio de aves mortas sob efeito dos tratamentos contendo farelo de amendoim com ou sem a presença de aluminosilicatos nos diferentes períodos de vida das aves em relação ao controle. Esses resultados concordam com os obtidos por LANZA et al. (1980).

De acordo com KUBENA et al. (1990), foi observado aumento de mortalidade quando as aves consumiam ração contaminada sem aluminosilicato de sódio hidratado. Este mesmo resultado, porém, não foi encontrado neste estudo quando as aves ingeriram ração contaminada com aluminosilicatos 1 ou 2.

A análise estatística dos valores referentes aos parâmetros sanguíneos em estudo é mostrada na TABELA 8. 
TABELA 6. Efeito dos tratamentos na conversão alimentar das aves em cada um dos períodos estudados.

\begin{tabular}{|c|c|c|c|c|c|c|c|}
\hline \multirow[t]{3}{*}{ TRATAMENTO } & \multicolumn{7}{|c|}{$\begin{array}{l}\text { CONVERSÃO ALIMENTAR (KG DE RAÇÃO/KG DE GANHO DE } \\
\text { PESO) }\end{array}$} \\
\hline & $0-7$ & 8-14 & $15-21$ & $22-28$ & $29-35$ & $36-42$ & $43-47$ \\
\hline & \multicolumn{7}{|c|}{ (dias de idade) } \\
\hline 1 & $1,49^{\circ}$ & $1,66^{\circ}$ & $1,73^{\mathrm{a}}$ & $1,98^{\mathrm{a}}$ & $2,10^{\circ}$ & $2,40^{a}$ & $2,43^{\mathrm{a}}$ \\
\hline 2 & $1,67^{\circ}$ & $1,93^{\mathrm{a}}$ & $1,72^{\circ}$ & $1,75^{\mathrm{a}}$ & $1,84^{\mathrm{a}}$ & $2,25^{a}$ & $2,47^{\mathrm{a}}$ \\
\hline 3 & $1,51^{\mathrm{a}}$ & $1,99^{a}$ & $1,76^{\mathrm{a}}$ & $1,80^{\circ}$ & $2,09^{\mathrm{a}}$ & $2,01^{a}$ & $2,64^{a}$ \\
\hline 4 & $1,39^{\circ}$ & $1,86^{\circ}$ & $1,79^{\circ}$ & $2,03^{\circ}$ & $1,92^{\mathrm{a}}$ & $2,25^{\mathrm{a}}$ & $2,36^{\circ}$ \\
\hline 5 & $1,77^{\mathrm{a}}$ & $1,95^{\mathrm{a}}$ & $1,70^{a}$ & $1,91^{\circ}$ & $1,96^{\circ}$ & $2,23^{\mathrm{a}}$ & $2,24^{\mathrm{a}}$ \\
\hline 6 & $1,48^{\mathrm{a}}$ & $2,35^{b}$ & $1,74^{\mathrm{a}}$ & $1,91^{\circ}$ & $2,02^{\mathrm{a}}$ & $1,93^{\circ}$ & $2,59^{\mathrm{a}}$ \\
\hline
\end{tabular}

Valores em uma mesma coluna com diferentes sobrescritos, diferem significalivamente do controle $(\mathrm{P}<0,05)$.

TABELA 7. Efeito dos tratamentos na mortalidade das aves em cada um dos períodos estudados.

\begin{tabular}{|c|c|c|c|c|c|c|c|}
\hline \multirow[t]{2}{*}{ TRATAMENTO } & \multicolumn{7}{|c|}{ MORTALIDADE (N MÉDIO DE AVES) } \\
\hline & $0-7$ & $8-14$ & $15-21$ & $22-28$ & $29-35$ & $36-42$ & $43-47$ \\
\hline \multicolumn{8}{|c|}{ (dias de idade) } \\
\hline 1 & $0,0^{\mathrm{a}}$ & $0,2^{\mathrm{a}}$ & $0,0^{a}$ & $0,8^{a}$ & $0,2^{\mathrm{a}}$ & $0,0^{a}$ & $0,0^{\mathrm{a}}$ \\
\hline 2 & $0,8^{a}$ & $0,2^{\circ}$ & $0,0^{a}$ & $1,0^{\mathrm{e}}$ & $0,0^{a}$ & $0,0^{\mathrm{a}}$ & $0,4^{\mathrm{a}}$ \\
\hline 3 & $0,6^{a}$ & $1,2^{\mathrm{n}}$ & $0,2^{\circ}$ & $1,2^{\mathrm{a}}$ & $0,0^{n}$ & $0,4^{\circ}$ & $0,2^{\circ}$ \\
\hline 4 & $0,2^{\mathrm{a}}$ & $0,6^{\circ}$ & $0,0^{\circ}$ & $0,2^{\mathrm{a}}$ & $0,0^{\circ}$ & $0,0^{\circ}$ & $0,8^{a}$ \\
\hline 5 & $0,0^{\mathrm{a}}$ & $0,0^{\circ}$ & $0,6^{\mathrm{b}}$ & $1,4^{a}$ & $0,2^{\mathrm{a}}$ & $0,2^{\mathrm{B}}$ & $0,0^{\mathrm{a}}$ \\
\hline 6 & $0,4^{a}$ & $0,4^{n}$ & $0,2^{\mathrm{a}}$ & $1,0^{\circ}$ & $0,0^{\circ}$ & $0,4^{\circ}$ & $0,4^{a}$ \\
\hline
\end{tabular}

Valores em uma mesma coluna com diferentes sobrescritos, diferem significativamente do controle $(\mathrm{P}<0,05)$.

Os resultados indicados na TABELA 8 mostram que os niveis de bilirrubina, fosfatase alcalina, gama-glutamil transferase, capacidade total de ligação do ferro e tempo de protrombina não foram afetados de maneira significativa nos tratamentos que receberam ração com o farelo de amendoim com ou sem a presença dos aluminosilicatos quando comparados com o controle. 
TABELA 8. Níveis dos parâmetros sanguíneos.

\begin{tabular}{cccccccc}
\hline \hline TRATAMENTO & \multicolumn{7}{c}{ PARÂMETROS SANGUÍNEOS } \\
\hline & PT & BIL & FA & TGO & GGT & CTLF & TP \\
\hline 1 & $\mathrm{~g} \%$ & $\mathrm{mg} \%$ & $\mathrm{U} / 1$ & $\mathrm{U} / \mathrm{ml}$ & $\mathrm{U} / 1$ & $\mathrm{mg} \%$ & $(\mathrm{~s})$ \\
\hline 2 & $3,04^{\mathrm{a}}$ & $0,95^{\mathrm{a}}$ & $474,4^{\mathrm{a}}$ & $95,8^{\mathrm{a}}$ & $21,80^{\mathrm{a}}$ & $163,75^{\mathrm{a}}$ & $94,40^{\mathrm{a}}$ \\
3 & $2,65^{\mathrm{a}}$ & $0,84^{\mathrm{a}}$ & $571,6^{\mathrm{a}}$ & $126,0^{\mathrm{b}}$ & $24,40^{\mathrm{a}}$ & $122,50^{\mathrm{a}}$ & $86,00^{\mathrm{a}}$ \\
4 & $2,39^{\mathrm{b}}$ & $0,76^{\mathrm{a}}$ & $556,2^{\mathrm{a}}$ & $135,8^{\mathrm{b}}$ & $23,20^{\mathrm{a}}$ & $190,60^{\mathrm{a}}$ & $128,25^{\mathrm{a}}$ \\
5 & $2,82^{\mathrm{a}}$ & $0,87^{\circ}$ & $499,0^{\mathrm{a}}$ & $107,6^{\mathrm{a}}$ & $21,80^{\mathrm{a}}$ & $159,50^{\mathrm{a}}$ & $95,25^{\mathrm{a}}$ \\
6 & $2,29^{\mathrm{b}}$ & $0,76^{\mathrm{a}}$ & $511,6^{\mathrm{a}}$ & $114,4^{\mathrm{a}}$ & $23,25^{\mathrm{a}}$ & $167,60^{\mathrm{a}}$ & $92,60^{\mathrm{a}}$ \\
\hline
\end{tabular}

Valores em uma mesma coluna com diferentes sobrescritos, diferem significativamente do controle $(\mathrm{P}<0,05)$.

OBS: 1) PT (proteína total), BIL (bilirrubina), FA(fosfatase alcalina), TGO (transaminase glutâmica oxalacética), GGT(gama-glutamil transferase), CTLF (capacidade total de ligação do ferro) e TP (tempo de protrombina).

Entretanto, o nível de proteína total foi diminuido significativamente pelos tratamentos 3 , 5 e 6 em relação ao controle, enquanto os níveis de transaminase glutâmica oxalacética aumentaram significativamente em relação ao controle nos tratamentos 2 e 3 .

As diferenças estatisticamente significativas observadas em alguns parâmetros sanguíneos referentes aos tratamentos das aves alimentadas com rações contendo aflatoxina ou aflatoxina mais alumino-silicatos, mostraram não ser estatisticamente diferentes em relação ao controle devido provavelmente a inadequação da coleta das amostras de sangue, do preparo destas para o envio ao laboratório para serem analisadas ou das técnicas utilizadas pelo laboratório, o qual era adequado somente as análises de sangue humano.

Um outro fator a ser considerado é o nível de toxicidade detectado neste trabalho, que foi inferior ao encontrado na literatura mas superior ao exigido como nível máximo de contaminação pela legislação brasileira que é de $\mathbf{5 0}$ ppb nas rações.
Os resultados encontrados neste trabalho referentes a redução do teor de proteína total concordam com os trabalhos de HARVEY et al. (1988a), HUFF et al. (1986a) e HUFF et al. (1988). Os resultados referentes ao aumento do nível de transaminase glutâmica oxalacética no soro das aves estão concordantes com os trabalhos de DALVI \& MACGOWAN (1984) e BABACHANDRAN \& RAMARKRISHNAN (1988).

Os resultados observados neste trabalho referentes a bilirrubina, fosfatase alcalina, gamaglutamil transferase, capacidade total de ligação do ferro e tempo de protrombina em aves tratadas com aflatoxina não foram significativamente diferentes em relação ao controle.

Entretanto, de acordo com a citação de LEHNINGER (1990), a bilirrubina ligada a albumina é transportada através do sangue para o figado e sendo o nível de albumina reduzido sob a ação da aflatoxina (HUFF et al. 1986a), acredita-se que o nível de bilirrubina de uma ave tenda a diminuir. Essa tendência foi observada no presente trabalho. 
GARLICH et al. (1973) relataram que a aflatoxina aumentou o nível da fosfatase alcalina no soro. Apesar das diferenças não serem significativas o presente trabalho mostrou um aumento da fosfatase alcalina em relação ao controle.

DOERR \& HAMILTON (1981) constataram prolongamento do tempo de protrombina. Esse aumento embora pequeno e não significativo foi verificado quando as aves ingeriram aflatoxina mais alumino-silicatos.

Os níveis da enzima GGT foram observados por KUBENA et al. (1990), que relataram que frangas alimentadas com rações contendo aflatoxina apresentaram aumento da atividade desta enzima. Foi observada neste trabalho uma tendência de pequena elevação apesar de não ter sido significativa em relação ao controle.

Os trabalhos de HARVEY et al. (1988b) indicaram redução progressiva da capacidade total de ligação do ferro. Foi observada também redução na CTLF no presente trabalho quando as aves ingeriram ração sem adição de aluminosilicatos e contendo $428,8 \mathrm{ppb}$ de aflatoxina B1. Entretanto, essa redução não foi significativa.

A diferença observada em relação ao nível de contaminação de aflatoxina nas rações gerou uma limitação no que se refere à análise estatística dos dados e interpretação dos resultados. Esta diferença pode ser explicada pela desuniformidade da toxina no farelo de amendoim em decorrência da desuniformidade da contaminação no grão, levando a uma maior concentração da toxina em algumas partículas, e, como existem diferenças de densidade de partículas, apesar da homogeneização do farelo de amendoim, ocorreu a formação de pontos de concentração de aflatoxina, o que acarretou diferentes teores de aflatoxina nas rações. Isto está de acordo com o trabalho de VELASCO \& MORRIS (1976), que relataram a variabilidade no teor de aflatoxina em materiais a serem analisados. Além disto, existiram interferências nas análises e quantificação da aflatoxina na ração, devido ao uso de produtos químicos na ração, tais como o antioxidante. Esse fato está de acordo com QURESHI (1991), que relatou que a análise tradicional de cromatografia, com posterior extração com solvente é questionável devido a distorção de resultados por antioxidantes e outros agentes químicos presentes no alimento. Além disso, TUNG et al. (1975) relataram que o método de cromatografia de PONS et al. (1966) não é um método preciso.

\section{CONCLUSÕES}

1. A aflatoxina atuou de maneira significativa, a fetando negativamente o desempenho das aves, no que se refere ao peso corporal médio, ganho de peso, consumo alimentar, conversão alimentar e mortalidade.

2. Foi observado efeito negativo nos parâmetros sanguíneos, proteína total e transaminase glutâmica oxalacética. Esse efeito não foi observado nos parâmetros sanguíneos bilirrubina, fosfatase alcalina, gama-glutamil transferase, capacidade total de ligação do ferro e tempo de protrombina. Foi observada uma pequena tendência de redução nos níveis da bilirrubina e capacidade total de ligação do ferro e aumento da fosfatase alcalina e gama-glutamil transferase em relação ao controle, porém não significativa. O tempo de protrombina se elevou de maneira não significativa quando as aves ingeriram aflatoxina mais alumino-silicatos.

3. Os produtos utilizados não conseguiram neutralizar os efeitos da aflatoxina. Embora a literatura relate resultado positivo do uso do alumino-silicato para controlar os efeitos da aflatoxina, não foi possivel verificar nem se existiu proteção parcial. A impossibilidade de resultados conclusivos a este respeito no presente trabalho, foi devido a inesperada desuniformidade nos níveis de contaminação com aflatoxina em relação aos diferentes tratamentos.

4. A impossiblidade de se detectar diferenças nos resultados entre o controle e os demais tratamentos referentes às análises sanguíneas, com exceção da proteína total e transaminase glutâmica oxalacética, sugere a necessidade da adoção de técnicas de análises mais específicas ou diferentes das que foram desenvolvidas. A literatura citou inúmeros trabalhos em que a aflatoxina atuou negativamente sobre os parâmetros sanguíneos em estudo.

5. O presente estudo sugere também a necessidade do desenvolvimento de metodologia de análise de aflatoxina que elimine a interferência dos produtos químicos utilizados na confecção das rações.

\section{REFERENCIAS BIBLIOGRÁFICAS}

BABACHANDRAN, C.; "RAMARKRISHNAN, R. Influence of dietary aflatoxin on certain senum enzyme levels in broiler chickens. Mycopathologia, Dordrecht, v.101, p.65-67, 1988. 
CARNAGHAN, R.B.A.; HARTLEY, R.D.; OKELLY, J. Toxicity and fluorescence propertie of the aflatoxins. Nature, London, v.200, n.4, p.911-1101, 1963.

COOMES, J.J.; FEUELL, A.J. Recommended procedures for detection and estimation of aflatoxin B1 in groundnuts and groundnut materials. Tropical Products Institute, London, n.13, p.1-24, 1965.

COTTIER, G.J.; MOORE, C.H.; DIENER, U.L.; DAVIS, N.D. Effect of continuous feeding of aflatoxin to chickens. Highlighs of Agriculture Research, Alabama, v.15, n.3, p.7, 1968.

DALVI, R.R. An overview of aflatoxicosis of poultry: its characteristics prevention and reduction. Veterinary Research Communications, Amsterdam, v.10, p.429-443, 1986.

DALVI, R.R.; MACGOWAN, C. Experimental induction of chronic aflatoxicosis in chickens by purified aflatoxin $B_{1}$ and its reversal by activated charcoal, phenobarbital, and reduced glutatione. Poultry Science, Champaign, v.63, n.3, p. 485-491, 1984.

DIENER, U.L.; COLE, R.J.; SANDERS, T.H.; PAYNE, G.A.; LEE, L.S.; KLICH, M.A. Epidemiology of aflatoxin formation by Arpergillus flavus. Annual Review of Phytopathology, Palo Alto, v.25, p.:249-270, 1987.

DOERR, J.A.; HAMILTON, P.B. Aflatoxicosis and intrinsic coagulation function in broiler chickens. Poultry Science, Champaign, v.60, n.7, p.1406-1411, 1981.

DOLLEAR, F.G.; MANN, G.E.; CODIFER, L.P.; GARDNER, H.K.; KOLTUN, S.P.; VIX, H.L.E. Elimination of aflatoxins from peanut meal. Journal of American Oil Chemical Sociation, Champaign, v.45, p.862-865, 1968.

GARLICH, J.D.; TUNG, T.; HAMITON, P.B. The effects of short term feeding of aflatoxin on $\mathrm{egg}$ production and some plasma constituents of the laying hen. Poultry Science, Champaign, v.52p.2206-2211, 1973.

GILL, J.L. ed. Design and analysis of experiments: in the animal and medical science. Ames, The lowa State University, 1986. v.1, p.409.

HARVEY, R.B.; CLARK, D.E.; HUFF, W.E.; KUBENA, L.F.; CORRIER, D.E.; PHILLIPS, T.D. Suppression of serum iron-binding capacity and bone marrow cellularity in pigs fed aflatoxin. Bulletin of
Enviromental Contamination and Toxicology, New York, v.40, p.576-583, 1988.

HARVEY, R.B.; HUFF, W.F.; KUBENA, L.F.; CORRIER, D.F.; PHILLIPS, T.D. Progression of aflatoxicosis in growing barrows. American Journal of Veterinary Research, Schaumburg, v.49, n.4, p.482-487, 1988.

HUFF, W.E.; HARVEY, R.B.; KUBENA, L.F. Toxic synergism between aflatoxin and T-2 Toxin in broiler chickens. Ponltry Science, Champaign, v.67, n.10, p.1418-1423, 1988.

HUFF, W.E.; KUBENA, L.F.; HARVEY, R.B.; CORRIER, D.E.; MOLLENHAUER, H.H. Progression of aflatoxicosis in broiler chickens. Poultry Science, Champaign, v.65, p.10, p.18911899, 1986.

HUFF, W.E.; KUBENA, L.F.; HARVEY, R.B.; HAGLER, W.M.; SWANSON, S.P.; PHILLIPS, T.D.; CREGER, C.R. Individual and combined effects of aflatoxin and deoxynivalenol (DON; Vomitoxin) in broiler chickens. Poultry Science, Champaign, v.65, n.7, p.1291-1298, 1986.

HUGGES, B.L.; BARNET, B.D.; JONES, J.E.; DICK, J.W.; NORRED, W.P. Safety of feeding aflatoxininactivated com to White Leghom layer-breeders. Poultry Science, Champaign, v.58, p.1202-1209, 1979.

KUBENA, L.F.; HARVEY, R.B.; HUFF, W.E.; CORRIER, D.E. Efficacy of a hydrated sodium calcium aluminosilicate to reduce the toxicity of aflatoxin and T-2 toxin. Poultry Science, Champaign, v.69, n.7, p.1078-1086, 1990.

LANZA, G.M.; WASHBURN, R.D.; WYATT, R.D.; EDWARDS, H.M. Strain variation on hematological response and iron absorption in chickens fed aflatoxin. Poultry Science, Champaign, p.59, n.7, p.1566, 1980.

LEHNINGER, A.L. Biossíntese de aminoácidos e nucleotidios. In: - Principias de biogufmica. 6 ed. São Paulo, Sarvier, 1990. p.437-458.

MERKLEY, J.W.; MAXWELL, R.J.; PHILLIPS, J.G.; HUFF, W.E. Hepatic fatty acid profiles in aflatoxinexposed broiler chickens. Poultry Science, Champaign, v.66, n.1, p.59-67, 1987.

NYATHI, C.B.; HASLER, J.A.; CHETSANGA, C.J. The effect of diet on carcinogenicity of aflatoxin $B 1$ in animals: a review. Zimbabwe Veterinary Journal, Harare, v.16, n.1/2, p.19-22, 1985. 
OSTROWSKI-MEISSNER, H.T. Effect of contamination of diets with aflatoxins on growing ducks and chickens. Tropical Animal and Health Production, Yucatan, v.15, p.161-168, 1983.

PHILLIPS, T.D.; KUBENA, L.F; HARVEY, R.B.; TAYLOR, D.R.; HEIDELBAUGH, N.D. Hydrated sodium calcium aluminosilicate: a high affinity sorbent for aflatoxin. Poultry Science, Champaign, v.67, p.243-247, 1988.

PONS JUNIOR, W.A.; CUCULLU, A.F.; LEE, L.S.; ROBERTSON, J.A.; FRANZ, A.O.; GOLDBLATT, L.A. Determination of aflatoxins in agricultural products: use of aqueous acetone for extraction. Journal of the ASSOCIATION OF OFFICIAL ANALYTICAL CHEMISTS, Chicago, v.49, n.3, p.554-662, 1966.

RAM, V.K.; RAO, G.D.; RAO, R.P. Effect of aflatoxin feeding and its withdrawal effect on the growth rate of broilers and layers under long tern feeding trial. Indian Veterinary Journal, Madras, v.65, p.113-116, 1988.
SAS INSTITUTE. The ANOVA procedure. In: SAS/stat guide: for personal computers. 6 ed. . Cary, 1987. cap.11, p.125-154.

SAS INSTITUTE. The GLM procedure. In: SAS/stat guide: for personal computers. $6 \mathrm{ed}$. Cary, 1987. cap. 20, p.549-640.

TUNG, T.; COOK, F.W.; WYATT, R.D.; HAMILTON, P.B. The anemia caused by aflatoxin. Poultry Science, Champaign, v.54, p.1962-1969, 1975.

VELASCO, J.; MORRIS, S.L. Use of water slurries in aflatoxin analysis. Joumal of Agricultural and Food Chemistry, Maryland, v.24, n.1, p.86-88, 1976.

QURESHI, A.A. What are safe mycotoxin levels ? Poultry International, Mount Morris, v.30, n.3. p.14-18, 1991 .

Trabalho enviado para publicação em 06.05.92 Trabalho aceito para publicação em 14.04 .93 